\title{
DyNamical Evolution of STEllar Clusters AND ASSOCIATIONS IN THE FIELD OF TIDAL FORCES OF THE GALAXY
}

\author{
T.S. Kozhanov \\ Astrophysical Institute of the Kazakh Academy of Sciences, \\ 480068 Alma-Ata, 68, Russia
}

\section{A BSTRACT}

The equations of motion of the star-members of the cluster averaged on the elliptic orbits are obtained. These equations take into account the tidal forces of the Galaxy. The generalization of the Lagrange-Jacobi equation and Sundman inequality for non-classical scheme of the many-body problems is revised. The dynamical evolution of the moment of inertia is studied.Some theorens which determine the type of the star motion in the cluster are formulated.

\section{INIRODUCT ION}

In the frameworks of a classical theory of the gravita$t$ ion some dynamical aspects of non-classical scheme of the $n$-body problem is investigated. Particularly the study of the evolution behaviour of the stellar clusters and associations in the gravitational field of the Galaxy belongs to this scheme. Let us consider the galactical systems stellar clusters and associations and assume that the center of mass of those objects moves as a material point on the Keplerian ellipses relatively to the center of the Galaxy. In such a approach the regular gravitational field of the Galaxy, which is taking into account the tidal force and the proper gravitational field of clusters and associations influence the individual members of steller clusters and associations.

According to [4], in such statement of the problem, the equations of the motion of the $i-t h$ member of a cluster in the pulsating coordinate system with the eccentric anomaly $E$ as an independent variable, have the following form: 


$$
\begin{aligned}
& m_{i}\left\{\frac{d^{2} \xi_{i}}{d E^{2}}+\frac{P e \sin E}{\left(1-e^{2}\right) r} \quad \frac{d \xi_{i}}{d E}-2 \frac{P}{\sqrt{1-e^{2} r}} \frac{d n_{i}}{d E}-\frac{3 P}{\left(1-e^{2}\right) r} \xi_{i}\right\} \\
& =\frac{p^{2}}{\operatorname{rc}^{2}\left(1-e^{2}\right)} \frac{\partial \tilde{\Omega}}{\partial \xi_{i}} \\
& m_{i}\left\{\frac{d^{2} \zeta_{i}}{d E^{2}}+\frac{p e \sin E}{\left(1-e^{2}\right) r} \frac{d \eta_{i}}{d E}+2 \frac{p}{\sqrt{1-e^{2} r}} \frac{d \xi_{i}}{d E}\right\}=\frac{p^{2}}{r c^{2}\left(1-e^{2}\right)} \frac{\partial \tilde{\Omega}}{\partial \eta_{i}} \\
& m_{i}\left\{\frac{d^{2} \zeta_{i}}{d E^{2}}+\frac{P e \sin E}{\left(1-e^{2}\right) r} \frac{d \zeta_{i}}{d E}+\left[\frac{P-r}{r^{2}\left(1-e^{2}\right)} p-\frac{\alpha P^{2}\left(1-e^{2}\right)}{c^{2}} r^{2}\right] \zeta_{i}\right\} \\
& =\frac{p^{2}}{r c^{2}\left(1-e^{2}\right)} \frac{\partial \tilde{\Omega}}{\partial} \frac{\partial}{\zeta}
\end{aligned}
$$

where $\tilde{\Omega}$ is the force function of the system in the pulsating coordinates and

$$
\alpha=\left(\frac{\partial^{2} \phi}{\partial z^{2}}\right)_{0}, r=\frac{p}{1-e^{2}}(1-e \cos E), 0 \leq e<1
$$

The relation between $E$ and $t$ is determined by Kepler's equation

$$
E-e \sin E=n(t-\tau), \quad n=\text { const. }
$$

To obtain maximum information about the dynamical evolution of stellar clusters, one can simplify the system of equations (1) introducing the averaging value of an arbitrary $2 \pi$ periodical function $f(\xi, \eta, \zeta, E)$ over the argument $E$, by using formula [2]

$$
\bar{f}=\frac{1}{2 \pi} \int_{0}^{2 \pi} f(E) d E
$$

The obtained averaged equations of motion posses the Jacobi int egral

$$
\mathrm{T}-\beta \tilde{\Omega}-\mathrm{AJ}=\mathrm{h}=\text { const },
$$

and the integral of angular momentum in $\xi, \eta, \zeta$ axes 


$$
L_{\zeta}=\sum_{i}^{n} m_{i}\left(\xi_{i} n_{i}-n_{i} \xi_{i}^{\prime}\right)+\sum_{i}^{n} m_{i}\left(\xi_{i}^{2}+n_{i}^{2}\right), \xi_{i}^{j}=\frac{d \xi_{i}}{d E},
$$

where

$$
\begin{aligned}
B & =\frac{p}{c^{2} \sqrt{1-e^{2}}}, A=\frac{3 q_{\xi}}{2 \sqrt{1-e^{2}}}+\frac{p^{4}\left(2+e^{2}\right) q_{\zeta}}{4 c^{2}\left(1-e^{2}\right)}, \\
J_{\xi} & =q_{\xi} J, J_{n}=q_{n} J, J_{\zeta}=q_{\zeta} J, J=\sum_{i}^{n} m_{i}\left(\xi_{i}^{2}+\eta_{i}^{2}+\zeta_{i}^{2}\right) .
\end{aligned}
$$

On the basis of the integrals mentioned above the LagrangeJacobi equation, where we take $E$ instead of the independent variable $t$, can be written as

$$
\frac{\mathrm{d}^{2} \mathrm{~J}}{\mathrm{dE}^{2}}+4 \gamma \mathrm{J}=2(\beta \tilde{\Omega}+2 \mathrm{H}), \quad \mathrm{H}=\mathrm{h}+\mathrm{L}_{\zeta}=\text { const. }
$$

where

$$
\gamma=A+q_{\xi}+q_{n}=\frac{2 \sqrt{1-e^{2}}-3}{2 \sqrt{1-e^{2}}} q_{\xi}+q_{\eta}-\frac{\alpha p^{4}\left(2+e^{2}\right)}{4 c^{2}\left(1-e^{2}\right)^{3}} q_{\zeta},
$$

$q_{\xi}, q_{\eta}$ and $q_{\zeta}$ are positive less then unity. They represent fiattening of the clusters and associations. The coefficient $\gamma$ may be positive or negative. If flattening of the cluster in $\xi$ and $n$ axes is larger than in $\zeta$ axis, that is, influence of the tidal force of the Galaxy is significantly stonger in $\xi$ and $n$ axes than in $\zeta$ axis, then $\gamma$ is positive. Otherwise $\gamma$ is negative.

In order to receive information about properties of the motion of cluster's and association's members, as considered in equation (5), we introduce according to $[5,7]$ "mean quadratic distance". for each member of the cluster. In this case the moment of inertia of the system $J$ and force function $\Omega$ are defined as

$$
J=\frac{M^{*}}{M} R^{2}, \tilde{\Omega}=\frac{G M^{*}}{R}, M^{*}=\sum_{i \leq j}^{n} m_{i} m_{j}, M=\underset{i}{\sum} m_{i}
$$

This approach may be applied in the investigation of the evolution of the stellar clusters and associations within a Galaxy: i.e. the components are of the same order. Such systems are called the multiple systens of the Trapesium type. 
According to [1] such objects are frequent in the Galaxy. As an example we can indicate the stellar cluster NGC-1981 Trapezium.

\section{EVOLUTION OF THE MOMENT OF INERTIA FOR THE HULTIPLE SYSTEMS OF THE TRAPEZIUM TYPE}

The Generalisation of the Lagrange-Jacobi equation (5) for non-classical schemes of the n-body problems is an effective way for studying the dynamics of the Galactical gravitating systems. Below, we give this equation for multiple stellar associations and clusters in the field of tidal force of the Galaxy. In such a case equation (5) together with the equations (6), has the form

$$
\frac{\mathrm{d}^{2} \mathrm{~J}}{\mathrm{dE}^{2}}+4 \gamma \mathrm{J}=\frac{\mathrm{b}}{\sqrt{J}}+2 \mathrm{H}
$$

The form of the equation (7) is similar to the form of equation previously presented in our papers $[5,6]$. The only difference is a physical interpretation of coefficients. Therefore, we shall use the same mathematical analysis for the study of equation (7) as in [6].

1. The equation (7) $\gamma>0$ is investigated. The equation(7) posseses the socalled "integral of the energy of inertia" [8]:

$$
C=\frac{1}{2}\left(\frac{\mathrm{dJ}}{\mathrm{dE}}\right)^{2}+2 \gamma \mathrm{J}^{2}-2 \mathrm{~b} / \overline{\mathrm{J}}-2 \mathrm{HJ}
$$

Line $C\left(J, J^{\prime}\right)=$ const. completely defines phase trajectories of systems of the phase plane $\left(J, J^{\prime}\right)$ and it is equal to

$$
C=\frac{1}{2}\left(\frac{\mathrm{dJ}}{\mathrm{dE}}\right)_{0}^{2}+2 \gamma \mathrm{J}_{0}-2 \mathrm{~b} / \bar{J}_{\mathrm{o}}-2 \mathrm{HJ},
$$

where index. "O" means, that the value $J$ and $\mathrm{dJ} / \mathrm{dE}$ must be taken at $E=0(t=\tau)$. Consequently, $C$ may be larger, equal or smaller than zero.

Introducing in (8) instead of $\mathrm{J}$ its value from equation (6) and separating a variable we have from the equation (8)

$$
\frac{1}{\sqrt{2}} \int_{\mathrm{R}_{1}} \int^{\mathrm{R}} \frac{\mathrm{dR}^{2}}{\sqrt{\mathrm{N}(\mathrm{R})}}=\mathrm{E},
$$

where pol ynomial $N(R)$ has the form

$$
N(R)=-2 \gamma R^{4}+2 b_{1} R+2 H_{1} R^{2}+C \geq 0,
$$


and

$$
\mathrm{b}_{1}=2 \mathrm{BG} \sqrt{\mathrm{M}^{*} \mathrm{M}}, \quad \mathrm{H}_{1}=\frac{\mathrm{M}}{\mathrm{M}^{*}} \mathrm{H}
$$

As shown in [7], the evolution behaviour of stellar clusters and associations under consideration depends on existence of the roots of the polynomial (11).

In the case at $\gamma>0$ polynomial (11) has two real and two complex roots, i.e. $R_{1}>0, R_{2}>0, R_{3}, R_{4}$ - complex. For mally the expression (7) may be treated as equation of motion for a test star under action of the force

$$
F(E)=-4 \gamma J^{2}+b J^{-\frac{1}{2}}+2 \mathrm{H}
$$

Consequently a test star on the semiplane $(J \geq 0, d J / d E)$ moves between $t$ wo circles with radii $R_{1}$ and $R_{2}$ the centres of which coincide with the stellar cluster center.Accordingly the moment of inertia of the system is bounded that is it pulsates bet ween $\mathrm{J}_{\min }$ and $\mathrm{J}_{\max }$.

2. Let us consider the case $\gamma<0$ and $H_{1}>0$. In this case elementary investigation of the roots of the equation (11) shows that in the phase semiplane $(J \geq 0, d J / d E)$, integral curves describe oscillations with increasing amplitude. Therefore stellar clusters and associations with positive total energy are in state of sway oscillations.

If in the initial moment of time $t=\tau(E=0)$ at $H>0$ it determines condition

$$
\frac{1}{2}\left(\frac{\mathrm{dJ}}{\mathrm{dE}}\right)_{0}^{2}<-2 \gamma \mathrm{J}_{0}^{2}+2 \mathrm{~b} / \bar{J}_{\mathrm{o}}+2 \mathrm{HJ}_{\mathrm{o}},
$$

then according to (9), integral energy of the inertia $C<0$. In this case polynomial (11) has the following possible combinations of the roots:

a) $\mathrm{R}_{1}>0, \mathrm{R}_{2}<0, \mathrm{R}_{3}, \mathrm{R}_{4}$ - complex. This corresponds to the previous case. In other words, we have stable systems. b) $R_{1}>0, R_{2}<R_{3}<R_{4}<0$. In this case there are three regions of real star motion: $R>R_{1}, R<R_{2}$ and $R<R_{3}<R_{4}$. Inside circle with radius $R_{2}$ cluster is contracted, outside circle with radius $R_{1}$ cluster ie expanded, but between concentric circles with radii $R_{3}$ and $R_{4}$ system oscillates.

THE SUNDMAN INEQUAL ITY FOR NON-CLASSICAL SCHEME OF THE MANYBODY PROBLEM AND THEOREMS

Sundman inequality plays a great role in analysis of the n-body problem to determine the property of the motion. Below 
the inequality would be generalized for non-classical scheme of gravitating problem for the many-body. The problem is an application of the Sundman inequality for the evolutional behaviour of the stellar complex in external gravitational field of the Galaxy.

According to [3] it is not difficult to write the following :

$$
2 T=K+\sum_{i}^{n} m_{i}\left(\frac{d \vec{\rho}_{i}}{d E}\right)^{2}
$$

where $T=\frac{1}{2} \sum_{i}^{n} m_{i}\left[\left(\frac{d \xi_{i}}{d E}\right)^{2}+\left(\frac{d \eta_{i}}{d E}\right)^{2}+\left(\frac{d \zeta}{d E}\right)^{2}\right]-$ total

kinetic energy of the clusters and associations, $\vec{\rho}_{1}-$ is radius vector of the $1-t h$ body in the axes relative to center of mass of the systems and

$$
\begin{aligned}
K=\sum_{i} \frac{m_{i}}{\rho_{i}^{2}}\left(\xi_{i} n_{i}^{\prime}-n_{i} \xi_{i}^{\prime}\right)^{2} & +\sum_{i} \frac{m_{i}}{\rho_{i}^{2}}\left(\eta_{i} \zeta_{i}^{\prime}-\zeta_{i} \eta_{i}^{\prime}\right)^{2} \\
& +\sum_{i} \frac{m_{i}}{2}\left(\zeta_{i} \xi_{i}^{\prime}-\xi_{i} \zeta_{i}^{\prime}\right)^{2}
\end{aligned}
$$

Using integral of the angular momentum of averaged system of equation (1) and applying rules of Lagrange multipliers determined conditional minimum of the sum (13):

$$
\mathrm{K} \geq \frac{\mathrm{L}^{2}}{\mathrm{~J}}-2 \mathrm{~L}_{\zeta}\left(\mathrm{q}_{\xi}+\mathrm{q}_{n}\right)+\left(\mathrm{q}_{\xi}+\mathrm{q}_{n}\right)^{2} \mathrm{~J}, \mathrm{~L}^{2}=\mathrm{L}_{\xi}^{2}+\mathrm{L}_{n}^{2}+\mathrm{L}_{\zeta}^{2} \text {, }
$$

Substituting expression (14) in (12), we have

$$
2 \mathrm{~T} \geq \frac{L^{2}}{J}-2 L_{\zeta}\left(q_{\xi}+q_{n}\right)+\left(q_{\xi}+q_{n}\right)^{2} J+\sum_{i=1}^{n} m_{i}\left(\frac{d \vec{p}}{d E}\right)^{2}
$$

Then, by using Jacobi integral (3), Lagrange-Jacobi equation (5) and inequality (15), we find

$$
\frac{\mathrm{d}^{2} \mathrm{~J}}{\mathrm{dE} E^{2}}+4 \gamma J-2 \tilde{\mathrm{H}} \geq \frac{\mathrm{L}^{2}}{J}+\sum_{i=1}^{n} m_{i}\left(\frac{\mathrm{d} \vec{\rho}_{i}}{\mathrm{dE}}\right)^{2},
$$

where

$$
\tilde{\gamma}=\frac{3}{2} A+\frac{1}{4}\left(q_{\xi}+q_{\eta}\right)\left(1-q_{\xi}-q_{\eta}\right), \tilde{H}=L_{\zeta}\left(2-q_{\xi}-q_{\eta}\right)+2 h
$$


On the other hand it is not difficult to show that, [3],

$$
\sum_{i=1}^{n} m_{i}\left(\frac{d \vec{\rho}}{d E}\right)^{2} \geq \frac{1}{4 J}\left(\frac{d J}{d E}\right)^{2}
$$

Consequently, from the inequality (16), we have

$$
\frac{\mathrm{d}^{2} \mathrm{~J}}{\mathrm{dE}}-\frac{1}{4 \mathrm{~J}}\left(\frac{\mathrm{dJ}}{\mathrm{dE}}\right)^{2}+4 \tilde{\gamma J}-2 \tilde{\mathrm{H}} \geq \frac{\mathrm{L}^{2}}{\mathrm{~J}}
$$

This inequality is a generalization of the Sundman inequality for stellar clusters in gravitating field of the Galaxy.

Integral of this inequality can be written in the form:

$$
\int_{J}^{J} \frac{d J}{\sqrt{-\frac{4}{3} \cdot \tilde{\gamma} J^{2}+8 \tilde{H} U+C_{1} / J-4 L^{2}}} \geq E, C_{1}=\text { const. }
$$

Investigation of above inequality will be studied later on.

THEOREM 1. If the Jacobi integral $h=0$ in (9) and at $t \rightarrow \infty(E \rightarrow \infty)$ satisfy condition

$$
\lim _{\substack{t \rightarrow \infty \\(E \rightarrow \infty)}} \frac{d}{d \bar{E}}\left(\frac{\vec{\rho}_{i}}{\sqrt{J}}\right) \Rightarrow 0,
$$

then the moment of the inertia of the system has unrestricted growt $h$ in

$$
J \sim \operatorname{sh}^{4 / 3}\left(3 \sqrt{\frac{A}{2}}+\frac{C}{2}\right), C=\text { const. }
$$

Proof: Corresponding to [9]

$$
\sum_{i=1}^{n} m_{i}\left[\frac{d}{d \bar{E}}\left(\frac{\vec{\rho}_{i}}{\sqrt{J}}\right)\right]^{2}=\frac{8 \mathrm{TJ}-(\mathrm{dJ} / \mathrm{d} E)^{2}}{J^{2}},
$$

since

$$
\lim _{\substack{t \rightarrow \infty \\(E \rightarrow \infty)}} \sum_{i=1}^{n} m_{i}\left[\frac{d}{d E}\left(\frac{\vec{\rho}_{i}}{\sqrt{J}}\right)\right]^{2} \Rightarrow 0,
$$

the

$$
8 \mathrm{TJ}-\left(\frac{\mathrm{dJ}}{\mathrm{dE}}\right)^{2} \Rightarrow 0
$$

From Jacobi integral we have 


$$
T=\beta \tilde{\Omega}+A J .
$$

Substituting this expression in (21) and using (C), we find

$$
\int_{0}^{R} \frac{\sqrt{\mathrm{R}} \mathrm{dR}}{\sqrt{\bar{B}^{2}+\mathrm{AR}^{3}}}=E, \tilde{B}=B G M,
$$

for which

$$
J \sim \operatorname{sh}^{4 / 3}\left(3 \sqrt{\frac{A}{2}}+\frac{C}{2}\right)
$$

THEOREM 2. If total mechanical energy of a stellar cluster in field of the tidal force of the Galaxy,

$$
\mathrm{T}-\beta \tilde{\Omega} \leq 0
$$

and Jacobi integral $h<0$, then $\forall t \geq 0$, function

$$
\rho_{\min }=\min \left\{\rho_{i j}\right\} \text {, }
$$

is satisfied following inequality

$$
\rho_{\min } \leq \sqrt{\frac{2\lfloor-h \mid}{A M}}
$$

Proof. From Jacobi integral it follows

$$
\mathrm{T}-\hat{B \tilde{\Omega}}=\mathrm{h}+\mathrm{AJ} \leq 0,
$$

or

$$
\frac{A}{M} \underset{i<j}{n} m_{i} m_{j} \rho_{i j}^{2} \leq|-h| .
$$

This inequality may be written

$$
\rho_{i j}^{2} \frac{A}{\bar{M}} \sum_{i<j}^{n} m_{i} m_{j} \leq|-h|,
$$

as well as

$$
\sum_{i<j}^{n} m_{i} m_{j} \leq \frac{M^{2}}{2}
$$

Then inequality (22) will be written as

$$
\rho_{\min } \leq \sqrt{\frac{2|-\mathrm{h}|}{\mathrm{AM}}}
$$


THEOREM 3. For any time $\tau>0$ and $\operatorname{small} \varepsilon>0, \exists \tau{ }^{\prime}$, $\tau,>\tau$, such that

$$
\rho_{\min }\left(\tau^{\prime}\right)<\frac{B M^{2}}{2\left[2|h|-2 L_{\zeta}-\varepsilon\right]}
$$

Proof: For any large time $\tau>0$, one can find $\tau^{\prime}>\tau$ such that

$$
B \tilde{\Omega}+2 \mathrm{H}>-\varepsilon
$$

If not, let for definite $\exists$ a value $\tau$ such that

$$
B \tilde{\Omega}+2 \mathrm{H} \leq-\varepsilon, \forall \mathrm{t}>\tau
$$

Let us solve Lagrange-Jacobi equation (5) in integral from $t_{0}$ to $t>\tau\left(t_{0}\right.$-correspoma $\left.E=0\right)$ for $\gamma>0$

$$
J \leq\left(J_{0}+\frac{\varepsilon}{2 \gamma}\right) \cos 2 \gamma E+\frac{(d J / d E)_{o}}{2 \gamma} \sin 2 \gamma E-\frac{\varepsilon}{2 \gamma} .
$$

From this equation we can obtain that for $E \rightarrow \infty$ the.moment of inertia periodically reaches to negative value (if $(\mathrm{dJ} / \mathrm{dE})_{0}<0$ or $\left.(d J / d E)_{0}<\varepsilon\right)$. But $I(E)$ must be positive. Hence, there existis $\tau^{\prime}>\tau\left(E^{\prime}>E\right)$ such that

$$
B \tilde{\Omega}+2 \mathrm{H}>-\varepsilon .
$$

Since $H=h+I_{\zeta}$, then

$$
B \tilde{\Omega}>-\varepsilon+2|\mathrm{~h}|-2 L_{\zeta}
$$

making this inequality stronger we obtain

$$
\rho_{\min }<\frac{\beta M^{2}}{2\left[2|h|-2 L_{\zeta}-\varepsilon\right]}
$$

\section{REF ER ENC ES}

[1] Ambartsumian, V.A.: 1954, in The Communication of the Byurakan Observatory, N4, p.3-35 (in Russian)

[2] Demin, V.G. and Evteev, V.P.: 1988, Eliptical Problems Three Eodies. "Donich", Dushanbe, p.133 (in Russian).

[3] Khilmi, G.F.: 1954, Qualitative Methods in the Many-Body Problem (in Russian). 
[4] Kozhanov, T.S.: 1990, in: Problems of Celestial Mech. and Stellar Systems. "Nauka": Alma-Ata. p.97-107 (in Russian).

[5] Kozhanov, T.S.: 1983 in: The Stellar Clusters and the Problems of Stellar Evolutions. Ural State University, Sverdlovsk, p.111-120 (in Russian).

[6] Kozhanov, T.S.: 1990, Kinamatics and Physics of the Celestial Bodies, V.6, N3, p.8-14 (Russian).

[7] Liu Tie and Sun, Y.S.: 1977/1989, Celes. Mech. V.44, NL-2, p. $117-134$.

[8] Osipkov, L.P. and Kozhanov, T.S.: Proc. of the Astrophys. Institute of the Kazahk Academy of Sciences. AlmaAta. V.45, p.32-47 (in Russian).

[ 9] Pollard, H.:1967, J.Math. Mech.V.17, 601-612. 